\section{Polymere Membranverbände Schmerztherapie bei Sportverletzungen}

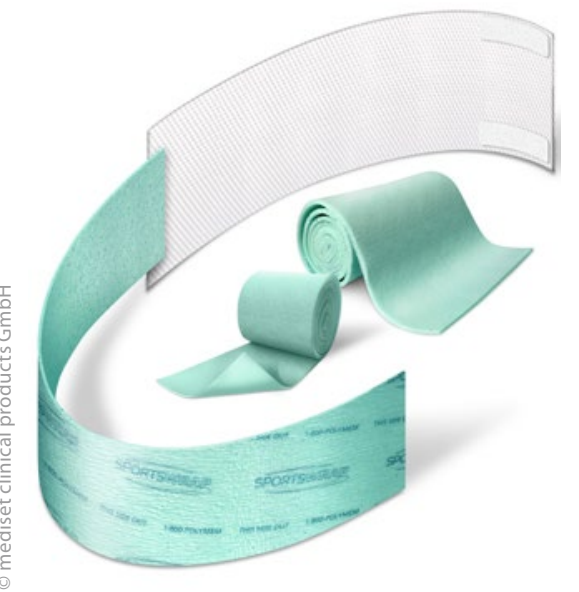

— Sportverletzungen, wie Stürze, Stöße oder ein Einknicken der Gelenke, sind oft mehr als nur schmerzhaft oder hinderlich beim Ausüben der sportlichen Tätigkeit. Eine Nichtbehandlung kann häufig zu erheblichen Folgeproblemen bis hin zum operativen Eingriff führen. Sich ausbreitende Entzündungen und Schwellungen sollten daher nicht leichtfertig betrachtet werden. Sportwrap von PolyMem ist ein Verband, der eigens für die Behandlung von Sportverletzungen entwickelt wurde. $\mathrm{Er}$ richtet sich speziell an stumpfe Verletzungen wie Prellungen, Zerrungen, Verstauchungen, Ödeme, Frakturen oder subkutane Blutungen. Das polymere Membranpolster kann direkt mittels eines integrierten Sekundärverbands angelegt werden kann. Sportswrap kann dabei als eine nicht-medikamentöse Schmerztherapie verstanden werden. Die Wirkstoffe in der Polymermembran hemmen die Wirkung von Nozizeptoren, die nach einer Gewebszerstörung oder Entzündung Schmerzinformationen transportieren. Somit wird der Ausbreitung von Entzündungen und Schwellungen entgegengewirkt. Zahlreiche Fallstudien haben bereits gezeigt, dass die Behandlung von stumpfen Verletzungen mit Polymermembranverbänden eine deutliche Senkung der sekundären mechanischen aber auch der thermischen Schmerzempfindlichkeit bewirken kann.

www.mediset.de

DKK 2016

\title{
Ernährungstherapie für Tumorpatienten
}

— Trinknahrung und Diätberatung können bei Tumorpatienten Lebensqualität und Therapietoleranz verbessern sowie die Komplikationsrate senken, so Prof. Dr. Matthias Pirlich, Chefarzt Innere Medizin an der Evangelischen Elisabeth Klinik, Berlin. Tumorpatienten mit einem Gewichtsverlust von mehr als $10 \%$ des Körpergewichtes haben eine signifikant kürzere Überlebenszeit als Patienten mit einem geringerem Gewichtsverlust $(p<0,001)$. Entsprechend sieht die S3-Leitlinie für Klinische Ernährung in der Onkologie ein Ernährungsscreening für alle onkologischen Patienten vor.

Den Vorteil einer Ernährungstherapie unterstreichen Ergebnisse von Lundholm et al., die über 300 Patienten evaluierten. Im Vergleich zur "besten Supportivtherapie" konnten Patienten mit einer fortgeschritte-

\section{Diabetes Mediendialog Digitalisierte Medizin}

— Digitalisierung ist ein Megatrend, der auch die Medizin in den nächsten Jahrzehnten massiv verändern wird. Besonders vorteilhaft ist die Anwendung dieser innovativen Technologien im Bereich des Diabetes Management. "Gerade in der Versorgung von Diabetikern bieten diese neuen Technologien wesentliche Vorteile, ja ein digitalisiertes Diabetes Management ist die unabdingbare Voraussetzung für eine personalisierte Diabetes-Therapie", erläuterte Dr. Christian Klepzig, Facharzt für Allgemeinmedizin in Offenbach/M. Die Vision, dass sich beim Besuch des Patienten in der Arztpraxis die Geräte automatisch mit dem Arztsystem verbinden und die Daten transferieren, so dass Analysegrafiken auf dem Display im Sprechzimmer erscheinen, wird allmählich Realität.

Im Moment wird der Arzt immer noch mit manuell geführten Tagebüchern konfrontiert. „Doch die Protokollierung der vom Diabetiker selbst gemessenen Werte schwankt in der Güte der Aufzeichnung, der Validität der Daten und der Lesbarkeit", so Klepzig. 60\% der Hypoglykämien werden auf diese Weise nicht dokumentiert. Eine strukturierte Analyse ist deshalb oft nicht nen Tumorerkrankung und einem Gewichtsverlust von einer eskalierenden Ernährungstherapie (Beratung und ggf. Trinknahrung bzw. supplementierende parenteraler Ernährung)" erheblich profitieren: Im Vergleich mit einer Standardversorgung zeigten sie ein signifikant längeres Überleben $(p<0,01)$ und eine bessere körperliche Leistungsfähigkeit. So kann Fortimel $^{\circledR}$ Compact 2.4, eine vollbilanzierte, hochkalorische Trinknahrung mit 2,4 kcal/ $\mathrm{ml}$ (300 kcal pro Flasche mit $125 \mathrm{ml}$ ) einen wertvollen Beitrag zur Verbesserung des Ernährungszustandes leisten.

(MW)

Satellitensymposium „Klinische Ernährung: Das unterschätzte Potential in der Krebstherapie"; 32. Deutscher Krebskongress (DKK), Berlin, 24.2.2016 (Veranstalter: Nutricia GmbH)

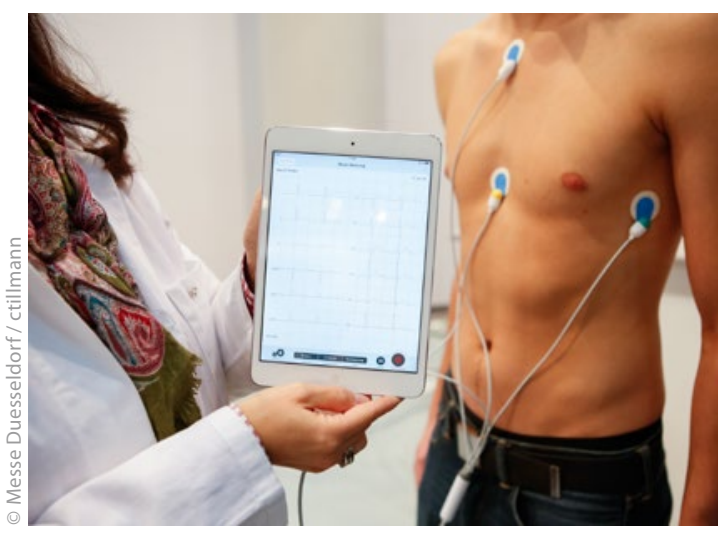

möglich. Die Visualisierung der Daten mit der Software "Smart pix" bietet wesentliche Vorteile für Arzt und Patient. Durch die schnellere Erfassung der Patientenprobleme werden ärztliche Gespräche effektiver. Um die Umsetzung von E-Health zu fördern, hat die AOK Hessen mit der Firma Roche Diagnostics jetzt einen integrierten Versorgungsvertrag abgeschlossen, nach dem die digitalisierte Auswertung der Blutzuckermesswerte besonders vergütet wird.

Diabetes Mediendialog „Digitale Medizin: E-Health, Personalisiertes Diabetes Management und die Zukunft der Diabetesversorgung", Schloss Hohenkammer, 4.3.2016 (Veranstalter: Roche Diagnostics) 